\title{
Logarithmic-type Scaling of the Collapse of Keller-Segel Equation
}

\author{
Sergey A. Dyachenko, Pavel M. Lushnikov and Natalia Vladimirova \\ Department of Mathematics and Statistics, University of New Mexico, Albuquerque, NM 87131, USA
}

\begin{abstract}
Keller-Segel equation (KS) is a parabolic-elliptic system of partial differential equations with applications to bacterial aggregation and collapse of self-gravitating gas of brownian particles. KS has striking qualitative similarities with nonlinear Schrodinger equation (NLS) including critical collapse (finite time point-wise singularity) in two dimensions. The self-similar solutions near blow up point are studied for KS in two dimensions together with time dependence of these solutions. We found logarithmic-type modifications to $\left(t_{0}-t\right)^{1 / 2}$ scaling law of self-similar solution in qualitative analogy with $\log -\log$ modification for NLS. We found very good agreement between the direct numerical simulations of KS and the analytical results obtained by developing a perturbation theory for logarithmic-type modifications. It suggests that log-log modification in NLS also could be verified in a similar way.
\end{abstract}

Keywords: Collapse and formation of singularities, Logarithmic modification of self-similar solution, Chemotaxis, Cellular aggregation, Self-gravitating Brownian particles

PACS: 05.45.-a, 87.18.Ed, 42.65.Jx, 87.18.Hf, 05.40.-a, 03.75.Kk

A Keller-Segel model (see e.g. [1-16] and references therein):

$$
\begin{aligned}
\partial_{t} \rho & =D \nabla^{2} \rho-\nabla[k \rho \nabla c], \\
\partial_{t} c & =D_{c} \nabla^{2} c+\alpha \rho,
\end{aligned}
$$

was derived for macroscopically averaged dynamics of bacteria with the bacterial density $\rho(\mathbf{r}, t)$ and the chemoattractant concentration $c(\mathbf{r}, t)$ at spatial point $\mathbf{r}$ and time $t$. Bacteria and biological cells often communicate through chemotaxis, when bacteria move towards chemical gradient of substance called chemoattractant. Bacteria can also secrete the same chemoattractant which creates nonlocal attraction between them through secretion, diffusion and detection of chemoattractant by other bacteria of the same type. Bacteria are self-propelled and without chemotactic clue center of mass of each bacteria typically experiences random walk. That random walk is described in (1) by the the diffusion coefficient $D$. Chemoattractant also diffuses with the diffusion coefficient $D_{c}$. Motion of bacterial colonies is thus affected by permanent competition of random-walk-based diffusion and chemotaxis-based attraction. Also the coefficient $k>0$ characterize strength of chemotaxis, and $\alpha$ is the production rate of chemoattractant by bacteria.

Typically diffusion of a chemical is much faster than cell diffusion, i.e. $D / D_{c} \ll 1$. E.g., $D / D_{c} \sim 1 / 40-1 / 400$ for the cellular slime mold Dictyostelium [17], and $D / D_{c} \sim 1 / 30$ for microglia cells and neutrophils [18, 19]. Thus Eq. (1) evolves on a much smaller time scale than Eq. (1) and one can neglect time derivative in (1) which gives a reduced Keller-Segel equation (RKS)

$$
\begin{aligned}
\partial_{t} \rho & =\nabla^{2} \rho-\nabla[\rho \nabla c], \\
\nabla^{2} c & =-\rho,
\end{aligned}
$$

where we assumed that $D, D_{c}, \alpha$ and $k$ are constants and rewrote all variables in dimensionless form as $t \rightarrow t_{0} t$, $r \rightarrow t_{0}^{1 / 2} D^{1 / 2} r, \rho \rightarrow\left(D_{c} / t_{0} \alpha k\right) \rho, c \rightarrow(D / k) c$ and $t_{0}$ is a typical timescale of the dynamics of $\rho$ in Eq. (1).

Eq. (2) also describes a dynamics of a gas of self-gravitating Brownian particles which has applications in astrophysics [10]. In that case the second Eq. in (2) is a Poisson equation for the gravitational potential $-c$ while $\rho$ is the gas density (all units are dimensionless). The first Eq. in (2) is a Smoluchowski equation for $\rho$ in the gravitational potential $-c$. Below we refer to $\rho$ and $c$ as the density of bacteria and the concentration of chemoattractant, respectively, but all results below are equally true for a gravitational collapse of the gas of self-gravitating Brownian particles.

In dimension one $(\mathrm{D}=1)$ the solution of RKS exist globally in time while for $D>1$ (e.g. in dimensions two and three ) the finite time singularity is possible [6] provided bacterial density is large enough. E.g. for $D=2$ finite time 
singularity occurs for $N>4$, where $N=\int \rho d \mathbf{r} /(2 \pi)$ is the total number of bacteria (in rescaled units) [20]. Below we consider case $D=2$ only.

Formation of singularity in a finite time (blow up) is a quite general phenomenon in many nonlinear systems including self-focusing in nonlinear optics, plasmas, hydrodynamics, and collapse of Bose-Einstein condensate [21]. Because of the conservation of $N$ the finite time singularity (blow up) occurs at a spatial point. When blow up is accompanied by a dramatic contraction of the spatial extent of solution when it is called it by collapse [21]. Collapse typically occurs when there is self-attraction in a nonlinear system. Exactly such scenario occurs for RKS. Collapse in RKS corresponds to the aggregation of bacterial colonies in biological application and gravitational collapse for self-gravitating Brownian particles.

If density of bacteria is initially low then diffusion typically exceeds attraction and density remains low. E.g., a typical time scale for evolution of the a low density bacteria Escherichia coli distribution in a petri dish is about a day [1] (see Figure 3A in Ref. [1]). If initial density is not very small so that attraction wins over diffusion then bacteria experience aggregation (see Figure 3B in Ref. [1]). A typical time scale of such aggregation in experiment on Escherichia coli [1] is several minutes so it has an explosive character compare to evolution of bacteria outside of aggregation area. Here we study how aggregation occurs from moderate initial bacterial density (below we give exact criterion for formation of aggregation). Aggregation corresponds to the collapse of bacterial density in the KellerSegel model (1). Aggregation is a first step to a formation of multicellular organisms and quite important in biological applications [7]. Near singularity the Keller-Segel model is not applicable when typical distance between bacteria is about size of bacteria. In that regime modification of the Keller-Segel equation was derived from microscopic stochastic dynamics of bacteria which prevents collapse due to excluded volume constraint (different bacteria cannot occupy the same volume) $[22,23,24]$. Here however the original Keller-Segel model without regularization is considered.

The main result of this work is that solution of RKS (2) in a spatial dimension $D=2$ near collapse time $t_{0}$ in neighborhood of spatial location of collapse $r=0$ has the following cylindrically-symmetrical self-similar form

$$
\begin{aligned}
\rho & =\frac{1}{L(t)^{2}} \frac{8}{\left(1+y^{2}\right)^{2}}, \\
c & =-2 \ln \left(1+y^{2}\right), \\
y & =\frac{r}{L(t)}
\end{aligned}
$$

where $L(t) \equiv L$ is the time-dependent spatial width of solution (below we often omit argument of $L$ for brevity) given by

$$
\begin{aligned}
L(t) & =2 e^{-\frac{2+\gamma}{2}} \sqrt{t_{0}-t} \exp \left\{-\sqrt{-\frac{\ln \beta\left(t_{0}-t\right)}{2}}+\frac{-1+b \ln [x-\tilde{M}])}{2 x}\right. \\
& \left.+\frac{-1+2 b}{4 x^{2}}+\frac{7+3 b\left(-2-2 \gamma-2[1+b] \ln [x-\tilde{M}]+b \ln ^{2}[x-\tilde{M}]+2 \ln 2\right)}{12 x^{3}}+O\left(\frac{1}{x^{3}}\right)\right\} \\
x & =\sqrt{-2 \ln \beta\left(t_{0}-t\right)}, \\
\tilde{M} & =-2-\gamma+\ln 2, \\
b & =\frac{\pi^{2}}{3}-2 \ln ^{2} 2+4 \ln 2+\gamma(-4-2 \gamma+4 \ln 2), \\
\beta & =2 \exp \left\{2 l^{*}-\frac{\tilde{M}^{2}}{2}\right\}, \\
l^{*} & =-\ln L_{1}-\frac{1}{4} \ln ^{2} a_{1}+\frac{\tilde{M}+1}{2} \ln a_{1}-\frac{b}{2}\left(\ln \ln \frac{1}{a_{1}}+\frac{1}{\ln \frac{1}{a_{1}}}\right), \\
L_{1} & =L\left(t_{1}\right), \quad a_{1}=a\left(t_{1}\right)
\end{aligned}
$$

Here $\gamma=0.577216 \ldots$ is the Euler's constant and $t_{1}$ is any moment of time when solution has converged to self-similar form after short initial transient.

For $\left(t-t_{0}\right) \ll 1$ one can replace (4) by the leading order term as follows

$$
L(t)=2 e^{-\frac{2+\gamma}{2}} \sqrt{t_{0}-t} e^{-\sqrt{-\frac{\ln \left(t_{0}-t\right)}{2}}} .
$$




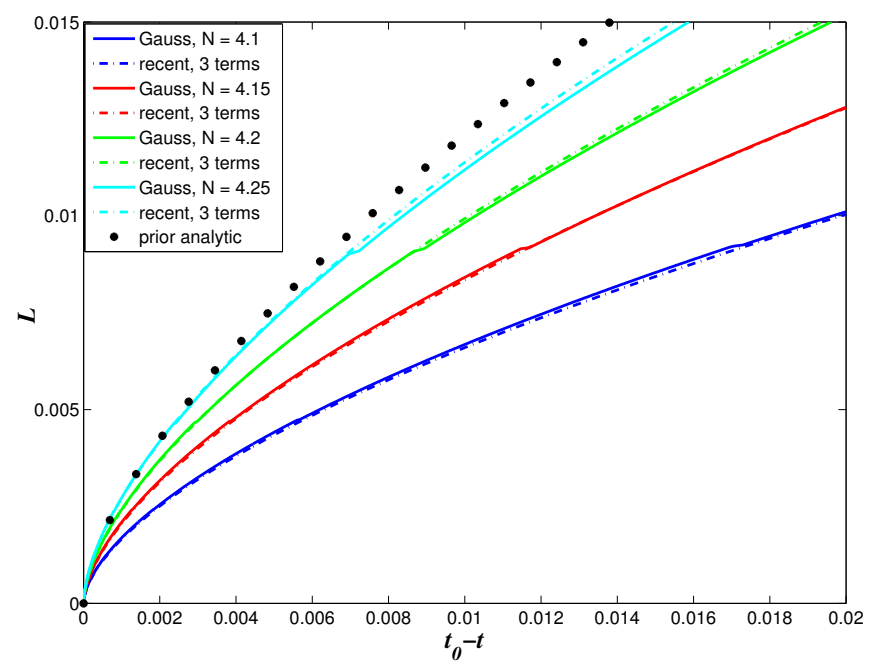

FIGURE 1. $L(t)$ dependence obtained from the direct numerical simulations of RKS (solid lines), given by the scaling (5) (dotted line) and given by the scaling (4) (dashed-dotted lines) for different values of $N$. The number of bacteria is defined as $N=\int \rho d \mathbf{r} /(2 \pi)$.

The scaling (5) was obtained in Ref. [9] using a formal matched asymptotic expansion and in Ref. [14] using the perturbation theory about the self-similar solution (3). Both scalings (4) and (5) have form $\propto \sqrt{t_{0}-t}$ with logarithmictype modifications. The scaling (5), while being asymptotically correct, requires extremely small values of $t-t_{0}$ for its applicability. We found from (4) and (5), that to quantitatively verify (5) in comparison with numerics, it is necessary to go for unrealistically small values of $L(t)$ (about $L(t) \sim 10^{-1000}$ or even much smaller). Instead we develop a perturbation theory which goes much beyond the accuracy of the equation (5) and results in the scaling (4). Figure 1 shows $L(t)$ from the the direct numerical simulations of RKS (solid lines represent simulations with different values of $N$ ), dotted line is given by (5) and dashed-dotted lines correspond to (4) with different values of $N$. It is seen that the agreement between numerical simulations and (4) is very good. But agreement between numerical simulations and (5) is quite poor. In addition, (5) is independent on $N$ which does not agree with the numerical simulations. That dependence on $N$ is expected for disappear for $L(t) \sim 10^{-1000}$ when both scalings (4) and (5) collapse to a single curve but simulations for such small $L$ would be quite challenging.

For numerical simulations we use the fact that near collapse the solution of RKS converges to the cylindricallysymmetric solution $\rho(\mathbf{r}, t)=\rho(r, t)$ [6], where $r=|\mathbf{r}|$. In that case it is convenient to introduce a new dependent variable $m$ as follows

$$
m(r, t)=\frac{1}{2 \pi} \int_{\left|r^{\prime}\right| \leq r} \rho\left(\mathbf{r}^{\prime}, t\right) d \mathbf{r}^{\prime},
$$

which allows [5] to rewrite RKS as a closed equation for $m$ :

$$
\partial_{t} m=r \partial_{r} r^{-1} \partial_{r} m+r^{-1} m \partial_{r} m .
$$

$m(r, t)$ in (7) has a meaning of a number of bacteria inside a circle of radius $r$. Boundary condition for $m$ at $r \rightarrow \infty$ is simply related to the total number of bacteria: $\left.m\right|_{r=\infty}=N$. In contrast to RKS, (7) is the partial differential equation for $m$. This simplification is possible for cylindrically-symmetric solutions of RKS only. We used (7) for simulations of RKS.

Small jumps in numerical values of $L(t)$ (seen in solid lines in Figure 1) result from the second order numerical differentiation necessary to extract $L(t)$ from the $m(r, t)$. We need that differentiation to determine $\rho(0, t)$ from $m(r, t)$ as follows: $\rho(0, t)=\left.\partial_{r}^{2} m(r, t)\right|_{r=0}$. After that $L(t)$ is obtained from (3) as $L(t)=[8 / \rho(0, t)]^{1 / 2}$. In simulation of (7) we use the numerical scheme with the adaptive mesh refinement which is the fourth order accurate in $r$. The small jumps in $L(t)$ is the result of the postprocessing of $m(r, t)$ data and they do not influence the accuracy of the numerical simulations of (7). We also performed simulations on a uniform spatial grid using pseudospectral Fourier method to 
evaluate the spatial derivatives in (7). The size of the grid was decreased using the trigonometric interpolation to the new grid when the current grid spacing was not enough to resolve Fourier spectrum beyond round-off error. That is we decreased grid spacing with the decrease of $L(t)$ to keep the spectral accuracy of simulations. Respectively, time step was also decreased. That second type of simulations agreed with the results of the adaptive mesh refinement simulations with high precision.

The self-similar scaling (3) has striking similarities with critical collapse in a two dimensional nonlinear Schrödinger equation (NLS) [25-32]

$$
i \partial_{t} \psi+\nabla^{2} \psi+|\psi|^{2} \psi=0 .
$$

E.g., collapsing solution of NLS (8) has a form of a rescaled ground state soliton solution of NLS while (3) has a form of a rescaled steady state solution of (2). Total number of bacteria in a self-similar solution (3) $N=\int \rho d \mathbf{r} /(2 \pi)=4$ is conserved which is s similar to conservation of the total number of particles in collapsing NLS solution. Scaling of collapsing NLS solution has the same leading order form $\left(t_{0}-t\right)^{1 / 2}$ as in (4) but a scaling modification for NLS has a well-known $\log$ - $\log$ form $L(t)=(2 \pi)^{1 / 2}\left(t_{0}-t\right)^{1 / 2} /\left(\ln \left[-\ln \left(t_{0}-t\right)\right]\right)^{1 / 2}$ which is different from an exponentof-square-root-of-logarithm for (4). The logarithmic modifications in both RKS and NLS result from nearly exact balance between linear and nonlinear terms (respectively balance between diffusion and attraction for RKS and balance between dispersion and nonlinear attraction in NLS). The log-log scaling for NLS is quite challenging for numerical verification [30]. We suggest, that similar to the difference between the scalings (5) and (4) for RKS, the correct way of verification of the NLS log-log scaling would be to develop a regular perturbation theory beyond the log-log leading order result.

This work was partially supported by NSF grants DMS 0719895 and DMS 0807131.

\section{REFERENCES}

1. M.P. Brenner, L. Levitov, and E.O. Budrene. Biophys. J. 74, 1677 (1998).

2. C.S. Patlak. Bull. Math. Biophys. 15, 311 (1953).

3. E.F. Keller, and L.A. Segel. J. Theor. Biol. 26, 399 (1970).

4. W. Alt, J. Math. Biol. 9, 147 (1980).

5. M.A. Herrero, and J.J.L Velázquez. Math. Ann. 306, 583 (1996).

6. M.P. Brenner, P. Constantin, L.P. Kadanoff, A. Schenkel, and S.C. Venkataramani. Nonlinearity 121071 (1999).

7. E. Ben-Jacob, I. Cohen, and H. Levine. Adv. Phys. 49 4, 395 (2000).

8. M.D. Betterton, and M.P. Brenner. Phys. Rev. E 64, 061904 (2001).

9. J.J.L. Velázquez. SIAM J. Appl. Math. 62, 1581 (2002).

10. C. Sire, and P.H. Chavanis. Phys. Rev. E 66, 046133 (2002).

11. T. Hillen, and H.G.Othmer. SIAM J. Appl. Math. 62, 1222 (2002).

12. R. Erban, and H.G.Othmer. SIAM J. Appl. Math. 65, 361 (2004).

13. T. J. Newman, and R. Grima. Phys. Rev. E 70, 051916 (2004).

14. P. M. Lushnikov, Physics Letters A 374, 1678 (2010).

15. S. Dejak, P. Lushnikov, Y. Ovchinnikov, and I. Sigal, Submitted to Physica D (2010).

16. S.I. Dejak, P.M. Lushnikov, and I.M. Sigal. On blowup dynamics in the Keller-Segel model of chemotaxis. In preparation.

17. T. Hofer, J.A. Sherratt, and P. K. Maini, Physica D 85, 425 (1995).

18. M. Luca, A. Chavez-Ross, L. Edelstein-Keshet, and A. Mogilner, Bull. Math. Biol. 65, 693 (2003).

19. R. Grima, Phys. Rev. Lett. 95, 128103 (2005).

20. P. Biler, and W.A. Woyczinski. SIAM J. Appl. Math. 59, 845 (1998).

21. E.A. Kuznetsov, and V.E. Zakharov (Eds.) Wave Collapse (World Scientific Publishing Company, 2007).

22. M. Alber, N. Chen, T. Glimm, and P.M. Lushnikov. Phys. Rev. E. 73, 051901 (2006).

23. M. Alber, N. Chen, P. M. Lushnikov, and S. A. Newman. Physical Review Letters, 99, 168102 (2007).

24. P. M. Lushnikov, N. Chen, and M. Alber. Physical Review E, 78, 061904 (2008).

25. S. N. Vlasov, V. A. Petrishchev and V. I. Talanov, Izv. Vys. Uchebn. Zaved. Radiofizika 14, 1353 (1971) [Radiophys. Quantum Electron. 14, 1062 (1971)].

26. V.E. Zakharov, Zh. Eksp. Teor. Fiz. 62, 1745 (1972) [Sov. Phys. JETP 35, 908 (1972)].

27. G.M. Fraiman, Zh. Eksp. Teor. Fiz. 88, 390 (1985) [Sov. Phys. JETP, 61, 228 (1985)].

28. B.J. LeMesurier, G. Papanicolaou, C. Sulem, and P.L. Sulem. Physica D, 31, 78 (1988).

29. S. Dyachenko, A.C. Newell, A.Pushkarev, and V.E. Zakharov. Physica D 57, 96 (1992).

30. G. Fibich, and G. Papanicolaou. SIAM J. Appl. Math. 60, 183 (1999).

31. C. Sulem, and P.L. Sulem. Nonlinear Schrödinger Equations: Self-Focusing and Wave Collapse (World Scientific, 1999).

32. F. Merle, and P. Raphael. J. Amer. Math Soc. 19, 37 (2006). 